\title{
Nota sobre el polen de Sechium chinantlense Lira \& Chiang y Parasicyos dieterleae Lira \& Torres (Cucurbitaceae) ${ }^{1}$
}

Recientemente Alvarado et al. (1992) publicaron la descripción del polen de varias especies pertenecientes a géneros de la subtribu Sicyinae sensu Jeffrey (1990). En ese trabajo se hizo uso de las técnicas fenéticas para estudiar las características palinológicas encontradas, con lo cual se pudieron definir con cierta claridad los límites de algunos de estos géneros, como por ejemplo Sechium y Microsechium, constatándose con ello la importancia taxonómica que este tipo de características tienen en la familia Cucurbitaceae. Durante los últimos años se han descrito dos nuevas especies para géneros de la subtribu Sicyinae [Sechium chinantlense Lira \& Chiang (Lira y Chiang, 1992) y Parasicyos dieterleae Lira \& Torres (Lira y Torres, 1991)], y esta nota tiene por objeto dar a conocer la morfología de sus granos de polen.

Las muestras de polen fueron obtenidas de ejemplares de herbario representativos de cada una de estas dos especies, los cuales se hallan depositados en el herbario MEXU. Los granos de polen fueron procesados y sus características se describieron mediante las técnicas y procedimientos señalados por Alvarado et al. (1992). Se tomaron mediciones de 10-20 granos de polen para cada una de las siguientes caractéres: eje polar (P); eje ecuatorial (E), la mayor distancia entre los extremos de dos de los colpos, longitud de las espinas y grosor de la exina. A partir de los datos obtenidos para los tres primeros caracteres antes mencionados se calcularon la relación polar/ecuatorial (P/E) y el índice de área polar(IAP). Asi mismo, se cuantificó el numero de colpos y se describieron todos los caracteres cualitativos que fueron observados. Las figuras 1 y 2 fueron realizadas en un microcopio LEITZ-DIALUX bajo los objetivos $40 \mathrm{X}$ y $100 \mathrm{X}$ y usando película Kodak PlusX (125 ASA). Los materiales de respaldo (preparaciones y negativos) están depositados en las colecciones palinológicas del Laboratorio de Paleobotánica del Instituto Nacional de Antropología e Historia, México y del Herbario Nacional de México.

\footnotetext{
I Trabajo apoyado por la Internacional Board for Plant Genetic Resources (FAO/IBOGR), mediante el proyecto "Estudios Taxonómicos y Ecogeográficos de las Cucurbitaceae de Latinoamérica" a cargo del primer autor entre 1990 y 1992 en el Herbario Nacional de México.
} 


\section{Sechium chinantlense Lira \& Chiang}

Descripción Palinológica. Eumónada, isopolar, isodiamétrico; suboblato, $75.0-83.0(\mathrm{x}=79.0) \times 78.0-97.0(\mathrm{x}=90.0) \mathrm{m} ; \mathrm{P} / \mathrm{E}=0.87$; estefanocolpado, 8-10 colpos. IAP $=0.38-0.51(\mathrm{x}=0.43)$. Tectado, equinado. Espinas alargadas, 6.2-7.4 ( $\mathrm{x}$ $=6.6) \mathrm{m}$ de largo, de base más ancha que su ápice y éste agudo con una cavidad translúcida. Area interespinal con patrón microreticulado. Exina 3.7-4.1 m de grosor; sexina más gruesa que la nexina (2:1); contorno polar circular, contorno ecuatorial elíptico-circular. Ilustración. Figura 1.

Ejemplares de Respaldo. MEXICO. Oaxaca. J.I. Calzada 15043, J. Castrejón \& F. Concepción 183, 194, 212, R. Lira \& J.C. Soto 1185.

\section{Parasicyos dieterleae Lira \& Torres}

Descripción Palinológica. Eumónada, isopolar, radial; oblato-esferoidal, 72.6-84.9 $(\mathrm{x}=79.0) \times 78.6-96.2(\mathrm{x}=85.8) \mathrm{m} ; \mathrm{P} / \mathrm{E}=0.91$; estefanocolpado, 7-9 colpos. $\mathrm{IAP}=0.42-0.46(\mathrm{x}=0.44)$. Tectado, equinado. Espinas cónicas 3.6-5.5 $(\mathrm{x}=4.6) \mathrm{m}$ de largo, de base más ancha que el ápice y éste agudo y sin cavidad translúcida. Area interespinal con patrón microreticulado. Exina $2.8 \mathrm{~m}$ de grosor; sexina más gruesa que la nexina (2:1); contorno polar circular, contorno ecuatorial circular. Ilustración. Figura 2.

Ejemplares de Respaldo. MEXICO. Oaxaca. A. Garcia Mendoza 546, 1704 , M. Sousa et al. 5958, R. Torres 12318 .

Las características generales de los granos de polen de las dos especies estudiadas corresponden claramente con las consideradas por Jeffrey (1990) como típicas de los miembros de la subtribu Sicyinae (granos espinosos y colpados). En lo particular, el polen de Sechium chinantlense presenta rasgos que coinciden con los descritos por Alvarado et al. (1992) para las especies de Sechium, Frantzia y Polakowskia [= Sechium sensu Jeffrey (1978) y Lira y Chiang (1992)], aunque los granos, las espinas, y sobre todo la exina en $S$. chinantlense son de mayores dimensiones (ver cuadro 1). Así mismo, en el polen de S. chinantlense se presenta una mayor variación en el número de colpos y estos son de menor longitud, lo que tiene como consecuencia que el área apocolpial sea un poco más amplia. Por otra parte, el patrón microreticulado del área interespinal del polen de $S$. chinantlense, presenta lúmenes con un diámetro ligeramente mayor a lo que se observa en $S$. tacaco.

En cuanto al polen de Parasicyos dieterleae, aunque como puede verse en el cuadro 1, sus granos son claramente más grandes que los de la única otra especie conocida del género ( $P$. maculatus Dieterle en Alvarado et al., 1992), son muy similares a los de esta última, principalmente en cuanto al número de colpos, la longitud de las espinas y la ausencia en el ápice de éstas últimas de una cavidad translúcida. 

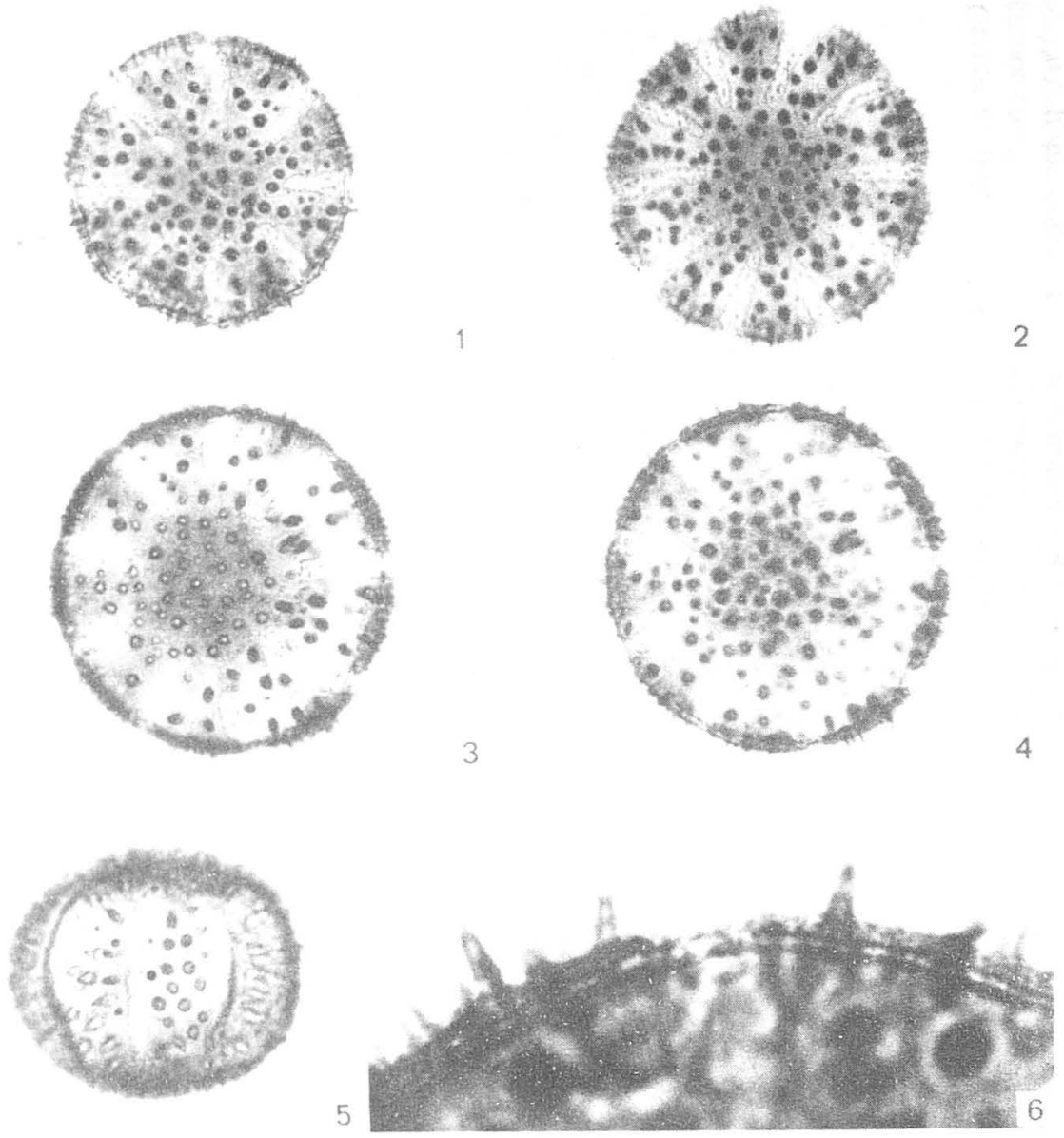

Fig. 1. Granos de polen de Sechium chinantlense Lira \& Chiang. 1-4) Vistas polares, en 1-3 cortes supra-ópticos, apreciándose granos con 8,9 y 10 colpos; 4, sección óptica. 5) Vista ecuatorial corte supra-óptica. 6) Detalle de las espinas y de la exina en sección óptica. Escala $=500 \mathrm{X}$. 

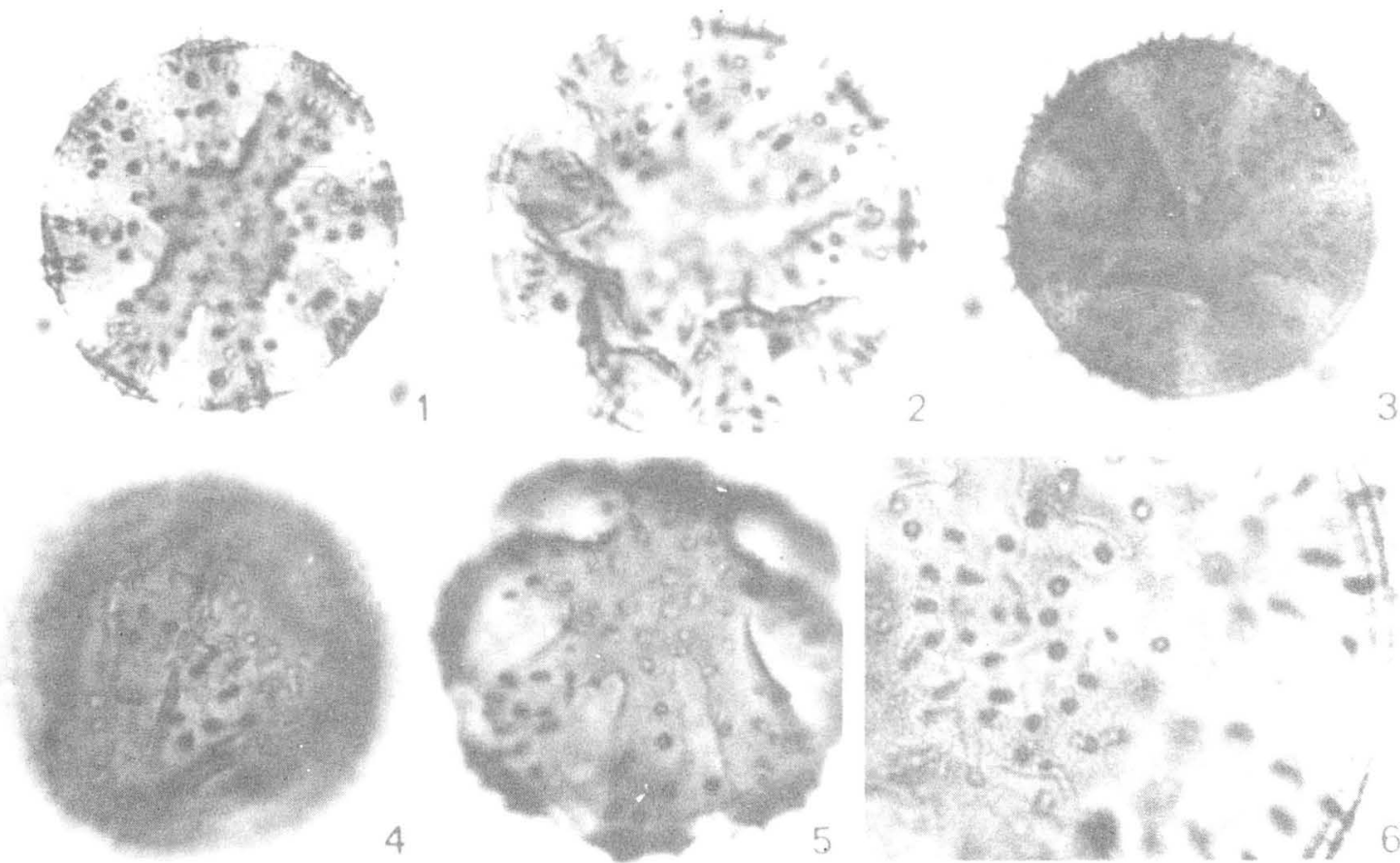

Fig. 2. Granos de polen de Parasicyos dieterleae Lira \& Torres. 1-3) Vistas polares en sección óptica, apreciándose granos con 8, 9 y 7 colpos respectivamente. 4) Vista ecuatorial corte supra-óptica. 5) Vista oblicua supra-óptica. 6) Vista polar, detalle en sección óptica. Escala $=500 \mathrm{X}$. 
Cuadrol. Comparación de las características palinológicas de Sechium chinantlense y Parasicyos dieterleae con las registradas por Alvarado et al. (1992) para las restantes especies de sus respectivos géneros. RPE = relación polar/ecuatorial; $\mathrm{IAP}=$ índice de área polar. $\mathrm{P}$ = presente, $\mathrm{A}=$ ausente. Los datos para las características ejes polar y ecuatorial, grosor de la exina, y el incluído entre paréntesis para el largo de las espinas, corresponden a valores promedio.

\begin{tabular}{|c|c|c|c|c|c|c|c|c|c|}
\hline Especies / Características del Polen & $\begin{array}{l}\text { Eje } \\
\text { Polar } \\
(\mu \mathrm{m})\end{array}$ & $\begin{array}{c}\text { Eje } \\
\text { Ecuatorial } \\
(\mu \mathrm{m})\end{array}$ & RPE & IAP & Forma & $\begin{array}{c}\text { Largo Espinas } \\
(\mu \mathrm{m})\end{array}$ & $\begin{array}{l}\text { Cavidad } \\
\text { Apical }\end{array}$ & $\begin{array}{c}\text { Grosor } \\
\text { Exina } \\
(\mu \mathrm{m})\end{array}$ & $\begin{array}{l}\text { No. de } \\
\text { Colpos }\end{array}$ \\
\hline Sechium edule (Jacq) Sw. (cultivada) & 66.8 & 90.4 & 0.73 & 0.60 & oblato & $1.3-6.7(3.4)$ & $\mathrm{P}$ & 2.3 & $9-11$ \\
\hline S. edule (silvestre) & 64.0 & 82.7 & 0.77 & 0.55 & suboblato & $4.9-7.2(6.0)$ & $\mathrm{P}$ & 2.5 & $8-9$ \\
\hline S. compositum (J.D. Smith) C. Jeffrey & 60.8 & 81.0 & 0.74 & 0.26 & oblato & $4.6-6.3(5.1)$ & $\mathrm{P}$ & 3.0 & $8-9$ \\
\hline S. hintonii (P.G. Wilson) C. Jeffrey & 52.6 & 68.8 & 0.76 & 0.36 & suboblato & $5.4-6.3(5.8)$ & $\mathrm{P}$ & 2.5 & $9-10$ \\
\hline S. tacaco (Pittier) C. Jeffrey & 78.6 & 87.6 & 0.89 & 0.34 & $\begin{array}{l}\text { oblato } \\
\text {-esferoidal }\end{array}$ & $6.1-7.2(6.5)$ & $\mathrm{P}$ & 3.0 & $8-9$ \\
\hline S. talamancense (Wunderlin) C. Jeffrey & 46.3 & 60.8 & 0.76 & 0.49 & suboblato & $3.6-8.1(5.5)$ & $\mathrm{P}$ & 3.5 & $8-9$ \\
\hline $\begin{array}{l}\text { S. panamense (Wunderlin) Lira } \\
\text { \& Chiang }\end{array}$ & 73.0 & 81.2 & 0.89 & 0.32 & $\begin{array}{l}\text { oblato } \\
\text {-esferoidal }\end{array}$ & $4.9-5.8(5.4)$ & $\mathrm{P}$ & 2.7 & $8-9$ \\
\hline S. pittieri (Cogn.) C. Jeffrey & 48.5 & 64.4 & 0.75 & 0.36 & suboblato & $5.6-7.1(6.4)$ & $\mathrm{P}$ & 2.8 & $8-9$ \\
\hline $\begin{array}{l}\text { S. venosum (L.D. Gómez) Lira } \\
\text { \& Chiang }\end{array}$ & 69.3 & 76.1 & 0.91 & 0.34 & $\begin{array}{l}\text { oblato } \\
\text {-esferoidal }\end{array}$ & $4.4-5.7(4.9)$ & $\mathrm{P}$ & 2.8 & $8-9$ \\
\hline S. villosum (Wunderlin) C. Jeffrey & 54.3 & 73.7 & 0.73 & 0.40 & oblato & $7.2-9.9(8.4)$ & $\mathrm{P}$ & 2.5 & $8-9$ \\
\hline Parasicyos maculatus Dieterle & 40.1 & 41.7 & 0.96 & 0.21 & $\begin{array}{l}\text { oblato } \\
\text {-esferoidal }\end{array}$ & $4.1-4.9(4.4)$ & A & 2.2 & $8-9$ \\
\hline Sechium chinantlense Lira \& Chiang & 79.0 & 90.0 & 0.87 & 0.43 & suboblato & $6.2-7.4(6.6)$ & $\mathrm{P}$ & 3.9 & $8-10$ \\
\hline Parasicyos dieterle Lira \& Torres & 79.0 & 85.8 & 0.91 & 0.44 & $\begin{array}{l}\text { oblato } \\
\text {-esferoidal }\end{array}$ & $3.6-5.5(4.5)$ & A & 2.8 & $7-9$ \\
\hline
\end{tabular}


La congruencia de las características palinológicas de estas especies y las de otras en sus respectivos géneros, es una evidencia más del valor de la morfología polínica en la taxonomía de la familia Cucurbitaceae.

\section{LITERATURA CITADA}

Alvarado. J. L., R. LiRa y J. Caballero. 1992. Palynological evidence for the generic delimitation of Sechium sensu lato (Cucurbitaceae) and its allies. Bull. Br. Mus. Nat. Hist. 22: 109-121.

JEFFreY, C. 1978. Further notes on Cucurbitaceae. IV. Some New World taxa. Kew Bull. 33 : 347-380.

JEFFREY, C. 1990. Appendix: An outline classification of the Cucurbitaceae. En: D. M. Bates, R.W. Robinson y C. Jeffrey (Edrs.). Biology and utilization of the Cucurbitaceae. Cornell Univ. Press, Ithaca, NY. pp. 449-463.

LiRA, R Y F. ChiAng. 1992. Two new combinations in Sechium (Cucurbitaceae) from Central America and a new species from Oaxaca, Mexico. Novon 2: 227-231.

LiRA, R. Y R. TORRES. 1991. Cuatro nuevos registros y una nueva especie de Cucurbitaceae para la flora de Oaxaca. Acta Bot. Mex. 16: 95-103.

\section{RAFAEL LIRA SAADE ${ }^{1}$, JOSÉ LUIS ALVARADO ${ }^{2}$ y JAVIER CASTREJÓN ${ }^{1}$}

${ }^{1}$ Herbario Nacional de México, Instituto de Biologia, Universidad Nacional Autónoma de México, Apdo. Postal 7)-233, Delegación Coyoacán, (0451(), México, D.F.

${ }^{2}$ Laboratorio de Paleobotánica, Instituto Nacional de Antropologia e Historia, Moneda 16. Centro, Delegación Cuauhtémoc, (06060), México, D.F. 